\title{
Evaluation of the anti-atherosclerosis effect of Shanhuaxiaozhi Formulation by combination of GC- MS-based metabolomics and TMT-based proteomics technology
}

\section{Xing Ren}

China Academy of Chinese Medical Sciences Xiyuan Hospital

Jing Yang

China Academy of Chinese Medical Sciences Xiyuan Hospital

\section{Baochen Zhu}

Beijing University of Chinese Medicine Affiliated Dongzhimen Hospital

Jianxun Ren

China Academy of Chinese Medical Sciences Xiyuan Hospital

\section{Shuai Shi}

China Academy of Chinese Medical Sciences Guanganmen Hospital

\section{Qiaoning Yang}

China Academy of Chinese Medical Sciences Xiyuan Hospital

\section{Wantong Zhang ( $\nabla$ wantong_zhang@hotmail.com )}

China Academy of Chinese Medical Sciences Xiyuan Hospital

\section{Rui Gao}

China Academy of Chinese Medical Sciences Xiyuan Hospital

\section{Research}

Keywords: Shanhuaxiaozhi formulation, Metabolomics, Proteomics, Atherosclerosis

Posted Date: September 22nd, 2021

DOI: https://doi.org/10.21203/rs.3.rs-903360/v1

License: (c) (i) This work is licensed under a Creative Commons Attribution 4.0 International License. Read Full License 


\section{Abstract \\ Objective}

Shanhuaxiaozhi formulation (SHXZF) is a traditional Chinese medicine preparation composed of Saffron, Crataegi Fructus, Chrysanthemi Flos, Tangerine Peel and Fructus Lycii. It is used in clinics for treating atherosclerosis with promising evidence of efficacy. Although some of the drugs in SHXZF have been reported to have significant therapeutic effects on atherosclerosis, the metabolic regulation and underlying mechanism of SHXZF during the remission of atherosclerosis are still unclear. This study aimed to integrate proteome and metabolome data sets for a holistic view of the molecular mechanisms of SHXZF in treating atherosclerosis.

\section{Methods}

Serum samples and aortic arch tissue from male $\mathrm{ApoE}^{-/-}$mice and $\mathrm{C} 57 \mathrm{BL} / 6 \mathrm{~L}$ mice were analyzed respectively using gas chromatography-mass spectrometry (GC-MS) based metabolomics technology and Tandem Mass Tags (TMT) based quantitative proteomics technology. Metabonomics and proteomics data were integrated to analyze the mechanism of SHXZF in the treatment of atherosclerosis.

\section{Results}

A total of 24 potential biomarkers and 70 differential proteins were identified. These substances were mainly involved in three biological pathways: the cAMP signaling pathway, the lipid metabolism pathway, and the myocardial contraction pathway.

\section{Conclusions}

The results suggested that SHXZF could effectively treat atherosclerosis, partially by regulating the above three metabolic pathways. The combination of proteomics and metabolomics provided a feasible method to uncover the underlying interventional effect and therapeutic mechanism of SHXZF on atherosclerosis.

\section{Introduction}

Atherosclerosis (AS) is a chronic inflammatory disease and characterized by the formation of lipidcontaining plaques in the vessel wall of arteries. It leads to narrowing or hardening of arteries, thereby restricting blood flow to the heart and eventually causing various cardiovascular and cerebrovascular events (Lee et al. 2018; Liu et al. 2020). As a multifactorial disease, prevention and treatment of AS are important, since with the development of the social economy and the aggravation of population aging, its incidence rate increases (Stavnsbo et al. 2018). About 17 to 18 million people die of atherosclerotic 
cardiovascular disease every year all over the world. It accounted for $31 \%$ of all deaths (Libby et al. 2019). Clinically, the AS patients are usually treated with statins, aspirin, nitroglycerin and other types of drugs (Wang et al. 2018). The typical treatments, like statins, in chemical medications against AS showed a substantial anti-AS and hypolipidemic effect. However, side effects, such as muscle pain, liver and kidney function damage, were commonly found and might limit clinical applications (Li et al. 2018). Therefore, many researchers are searching for a safer and alternative treatment for AS. In addition, some patients with mildly abnormal blood lipids refuse to take drugs such as statins, so there is a blank period of treatment.

Traditional Chinese medicine (TCM) has been extensively used in China and Eastern Asia for the treatment of AS. SHXZF is a TCM formula composed of five herbs and has obvious clinical effects in AS treatment Among them, saffron could lower blood pressure and serum cholesterol levels, therefore improving the clinical course of AS. Crocin is able to decrease the external cholesterol in macrophages and inhibit the uptake of oxidized low density lipoproteins (Stone et al. 2014). Studies have indicated that Crataegi Fructus and Chrysanthemi Flos may be potent phytochemical agents that inhibit formation of oxidized LDL (Leone et al. 2018). Similarly, the flavonoids in Tangerine Peel have antibacterial, antioxidant, and anti-tumor effects, which can lower cholesterol and prevent AS (Lin et al. 2014). Fructus Lycii,a traditional food and medicine in East Asia have become increasingly popular in Europe and North America in recent years. Antioxidant properties of Fructus Lycii have been detected in various in vitro and in vivo assays. The activity has been mainly attributed to the polysaccharides and has an active therapeutic effect on AS (Wu et al. 2021). At present, the efficacy of these drugs in the treatment of AS has been definitively verified, but the specific mechanism has not been revealed.

Omics techniques have been well used in diagnostics and experiment research. Metabolomics analysis, used to discover biomarkers and their perturbed pathways, can map the perturbations of early biochemical changes in diseases and result in an earlier intervention and insights into the therapeutic mechanisms (Luo et al. 2006). Proteomics method is being emerged as a powerful tool for characterizing differential proteins associated with differently physiological and pathological processes. Depending on this method, we can discover protein biomarkers for potentially therapeutic targets for drug discovery (Sun et al. 2013). At present, studies have used metabolomics or proteomics strategies to explore mechanism of traditional Chinese medicine compound on AS biological process (Colvin and Yeager 2015; Yang et al.2020). Therefore, in this work, a GC-MS based metabolomics technique was applied to discover biomarkers, and their perturbed pathways and changes after adiministration of SHXZF. Meanwhile a TMT based proteomics technique was utilized for searching the different proteins expressed and observing their variations after intervention with SHXZF. Finally, we integrated the data from both analyses to elucidate the SHXZF effects in protecting against AS.

\section{Materials And Methods}

\subsection{Materials and reagents}


Acetonitrile, Methanol, pyridine, $\mathrm{n}$-hexane, methoxylamine hydrochloride, and BSTFA were purchased from CNW Technologies GmbH (Düsseldorf, Germany), L-2-chlorophenylalanine was from Shanghai Hengchuang Biotechnology Co., Ltd. (Shanghai, China). Atorvastatin Calcium Tablets was purchased from Beijing JIALIN pharmaceutical Co., Ltd (Beijing, China). Herbs of SHXZF (Table 1) were purchased from the Xiyuan Hospital, China Academy of Chinese Medical Sciences (Beijing, China).

\subsection{Animal handling}

Eight-week male $\mathrm{ApoE}^{-/-}$mice and $\mathrm{C} 57 \mathrm{BL} / 6 \mathrm{~L}$ mice were purchased from Beijing Vital River Laboratory Animal Technology Co. Ltd (Beijing, China). 10 C57BL/6L mice were set to blank group and fed with normal diet. $30 \mathrm{ApoE}^{-/-}$mice were fed with a standard western diet (containing $20 \%$ protein, $50 \%$ carbohydrate and $21 \%$ fat, Beijing HFK bioscience Co., Ltd) to establish AS model. Then they were randomly divided into three groups: model group, Atorvastatin control group and SHXZF-treated group. The mice were fed adaptively for 3 days prior to the experiment. Mlce in SHXZF-treated group were administrated with SHXZF once a day in $0.1 \mathrm{~mL} / 10 \mathrm{~g}$ body weight for 8 weeks and blank group were given equal quantities of water. Mice in atorvastatin group were administrated with atorvastatin once a day in $2 \mathrm{mg} / \mathrm{kg}$ body weight.

\subsection{Oil red 0 staining section method and Pulse wave velocity (PWV) measurement}

\subsubsection{Oil red 0 staining section}

The fixed aortic root tissues was dehydrated with $10 \%, 20 \%$, and $30 \%$ sucrose solutions. Frozen sections $(6 \mu \mathrm{m}$ slice) were cut using a cryostat. Then soak the slices in $60 \%$ different propanol for 10 minutes, and dye with oil red staining solution for 10 minutes. Put the slices in $60 \%$ isopropanol to differentiate for 10 seconds, rinse with water after clarification, and mount a glass slide containing glycerin gelatin.

\subsubsection{PWV measurement}

The propagation time of the pulse wave from the aortic arch to the abdominal aorta is measured by the time delay between the upstroke (foot) of each pressure peak..Distance between the two measurement points is determined. Then divide the distance by the pulse wave travel time (in meters per second) to calculate the PWV. The travel time were taken by averaging at least 10 normal continuous cardiac cycles.

\subsection{Collection and preparation of biosamples}

$80 \mu \mathrm{L}$ of each serum sample was added to a 1.5-mL Eppendorf tube with $10 \mu \mathrm{L}$ of 2-chloro-lphenylalanine $(0.3 \mathrm{mg} / \mathrm{mL})$ dissolved in methanol as internal standard, and the tube was vortexed for $10 \mathrm{~s}$. Subsequently, $240 \mu \mathrm{L}$ of ice-cold mixture of methanol and acetonitrile $(2 / 1, \mathrm{v} / \mathrm{v})$ was added, vortexed for $1 \mathrm{~min}$, ultrasonicated for $5 \mathrm{~min}$, stored at $-20^{\circ} \mathrm{C}$ for $10 \mathrm{~min}$, centrifuged at $12000 \mathrm{rpm}, 4^{\circ} \mathrm{C}$ for $10 \mathrm{~min}$. An aliquot of the $150 \mu \mathrm{L}$ supernatant was taken for vacuum-dry at room temperature. Then added $80 \mu \mathrm{L}$ of $15 \mathrm{mg} / \mathrm{mL}$ methoxylamine hydrochloride in pyridine. The resultant mixture was vortexed vigorously for 
2 min and incubated at $37^{\circ} \mathrm{C}$ for 90 min. $80 \mu \mathrm{L}$ of BSTFA (with 1\% TMCS) and $20 \mu \mathrm{L}$-hexane was added, then vortexed vigorously for $2 \mathrm{~min}$ and derivatized at $70{ }^{\circ} \mathrm{C}$ for $60 \mathrm{~min}$.

\subsection{Metabolic profiling platform}

\subsubsection{Chromatography and mass spectrometry conditions}

Metabolic profiling analysis was performed using an GC-MS system with Agilent 7890B gas chromatography system and Agilent 5977A MSD system (Agilent Technologies Inc., CA, USA). A DB-5MS fused-silica capillary column (30 $\mathrm{m} \times 0.25 \mathrm{~mm} \times 0.25 \mu \mathrm{m}$, Agilent J \& W Scientific, Folsom, CA, USA) was utilized to separate the derivatives. Helium (>99.999\%) was used as the carrier gas with a flow rate of 1 $\mathrm{mL} / \mathrm{min}$. The injector temperature was $260^{\circ} \mathrm{C}$. Injection volume was $1 \mu \mathrm{L}$ by splitless mode. The initial oven temperature was $60{ }^{\circ} \mathrm{C}$, ramped to $125^{\circ} \mathrm{C}$ at a rate of $8{ }^{\circ} \mathrm{C} / \mathrm{min}$, to $210{ }^{\circ} \mathrm{C}$ at a rate of $5{ }^{\circ} \mathrm{C} / \mathrm{min} \otimes$ to $270{ }^{\circ} \mathrm{C}$ at a rate of $10{ }^{\circ} \mathrm{C} / \mathrm{min}$, to $305^{\circ} \mathrm{C}$ at a rate of $20^{\circ} \mathrm{C} / \mathrm{min}$, and finally held at $305^{\circ} \mathrm{C}$ for $5 \mathrm{~min}$. The temperature of MS quadrupole and ion source was 150 and $230{ }^{\circ} \mathrm{C}$, respectively. The collision energy was $70 \mathrm{eV}$.

\subsubsection{Data processing}

MD-DIAL software were used for data processing. Metabolites were annotated through LUG database (Untarget database of GC-MS from Lumingbio).

\subsubsection{Data analysis and biomarker identification}

Principle component analysis (PCA) an orthogonal partial least-squares-discriminant analysis ( OPLS-DA ) were performed to visualize the metabolic difference among experimental groups, after mean centering and unit variance scaling. The Hotelling's T2 region, shown as an ellipse in score plots of the models, defines the $95 \%$ confidence interval of the modeled variation. Variable importance in the projection (VIP) ranks the overall contribution of each variable to the OPLS-DA model, and those variables with VIP >1 are considered relevant for group discrimination. The differential metabolites were selected on the basis of the combination of a statistically significant threshold of VIP values obtained from the OPLS- DA model and $p$ values from a two-tailed Student's t-test on the normalized peak areas from different groups, where metabolites with VIP values larger than 1.0 and $p$ values less than 0.05 were considered as potential biomarkers.

\subsection{TMT-based proteomics analysis}

\subsubsection{Sample preparation and protein extraction}

Frozen samples of aortic arch tissue were transferred into low protein binding tubes (1.5ml Eppendorf) and lysed with $300 \mu \mathrm{L}$ lysis buffer supplemented with $1 \mathrm{mM}$ PMSF. Then the samples were further lysed with sonication. (80W, 3min). After sonication, the samples were centrifuged at $12000 \mathrm{~g}$ for $10 \mathrm{~min}$ to collect supernatants. Protein concentration was determined by BCA assay and aliquoted to store at $-80^{\circ} \mathrm{C}$. 


\subsubsection{Protein digestion and TMT labeling}

Add $25 \mathrm{~mm}$ DTT of corresponding volume into the protein solution. Then add the corresponding volume of iodoacetamide so that the final concentration is about $10 \mathrm{~mm}$. Subsequently, 6 times of the volume of precooled acetone in the above system to precipitate the protein. According to the amount of protein, add the corresponding volume of enzymolysis diluent (protein: enzyme $=50: 1(\mathrm{~m} / \mathrm{m}), 100 \mathrm{ug}$ of protein add $2 \mathrm{ug}$ of enzyme) to redissolve the protein precipitate. For TMT labelling, $88 \mu \mathrm{L}$ acetonitrile were added to TMT reagent vial. The centrifuged reagents were dissolved for $5 \mathrm{~min}$ and mixed for centrifugation for 2 times. Then $41 \mu \mathrm{L}$ of the TMT label reagent was added to each sample. Then incubated at room temperature for $1 \mathrm{~h}$. After that, $8 \mu \mathrm{L}$ of $5 \%$ hydroxylamine were added and incubated for $15 \mathrm{~min}$. The labeling peptides solutions were lyophilized and stored at $-80^{\circ} \mathrm{C}$.

\subsubsection{Liquid chromatography-mass spectrometry}

All analyses were performed by a QE mass spectrometer (Thermo, USA) equipped with an Easyspray source (Thermo, USA). Samples were loaded by a capillary trap column $(100 \mu \mathrm{m} \times 2 \mathrm{~cm}$, RP-C18, Thermo Fisher) and then separated by a capillary analytical column $(15 \mathrm{~cm} \times 75 \mu \mathrm{m}$, RP-C18, Thermo Fisher) on an EASY-nLCTM1200 system (Thermo, USA). The flow rate was $300 \mathrm{~nL} / \mathrm{min}$. The linear gradient was set as follow: $0 \sim 40 \mathrm{~min} \varangle 5-30 \% \mathrm{~B} \otimes 40 \sim 55 \mathrm{~min} \varangle 30-50 \% \mathrm{~B} \otimes 55 \sim 60 \mathrm{~min} \varangle 50-100 \%$ B. Full MS scans were acquired in the mass range of 300-1600 m/z with a mass resolution of 70000 and the AGC target value was set at 1e6. The 10 most intense peaks in MS were fragmented with higher-energy collisional dissociation (HCD) with collision energy of 28 . MS/MS spectra were obtained with a resolution of 35000 with an AGC target of $2 \mathrm{e} 5$ and a max injection time of $80 \mathrm{~ms}$. The dynamic exclusion was set for $15.0 \mathrm{~s}$ and run under positive mode.

\subsubsection{Protein identification and quantitative analysis}

The data were analyzed by Proteome Discoverer software 2.4 (Thermo Fisher Scientific, San Jose, CA, USA) and a database including 36,508 sequences (uniprot-reviewed-Mus musculus-2020_08). The search parameters were as follows: enzyme: trypsin; two missed cleavages allowed; fragment mass tolerance, $0.02 \mathrm{Da}$; mass values: monoisotopic; dynamic modification: Oxidation(M), Acetyl(N-term); peptide mass tolerance: 20 ppm; and fixed modifications: carbamidomethyl of cysteine. In addition, quality control was performed to determine if a reanalysis step was needed. The false discovery rate for both protein and peptide identification was set to be less than 0.01 . Fold changes $>1.5$ or $<0.75$ and $p<0.05$ were set as cut-off values to designate significant protein expression changes.

\subsubsection{Pathway enrichment and interaction network analysis}

Functional analysis of these genes expressed differentially was performed by DAVID (https://david.ncifcrf.gov).. Kyoto Encyclopedia of Genes and Genomes (KEGG) was utilized to analyze canonical pathways involved for those differentially expressed proteins. The protein-protein interaction network (PPI) was constructed using the online STRING database (https://string-db.org). 


\subsection{Statistical analysis}

The experimental results were presented as mean \pm SD and were compared using an 2-sample Student's $t$-test or a one-way ANOVA. Statistical difference was set at $p$ value $<0.05$.

\section{Results}

\subsection{Effect of SHXZF on pathomorphology of aortic root in ApoE-/- mice}

We evaluated the therapeutic impact of SHXZF on plaque development by histological examination of the cross-sectional lesions of the aortic sinus. At the same time, we can observe that the lipid and foam cells in model group stained by red oil 0 are more dense than other groups (Fig. 1A). As all of the roots of aorta cross-sections were not of the same size or diameter, we analyzed the percentage of plaque area relative to the vascular lumen area (Fig. 1B). In the MOD group, the percentage was $33.96 \pm 9.80$, which was significantly $(p<0.01)$ different from the average plaque area of the BLA group. Moreover, the percentage of plaque area to the vascular lumen area was significantly reduced in the aortic sections of the PCON $(15.39 \pm 6.40)$ and SHXZF $(15.01 \pm 12.20)$ groups. The percentages in the PCON and SHXZF groups were also significantly $(p<0.01)$ different from the average plaque area of the MOD group.

PWV has been used to assess the stiffness of large vessels. The PWV of the aortic was measured in each group of mice to obtain quantitative data of arterial stiffness(Fig. 1C). The PWV was $1.0 \pm 0.6 \mathrm{~m} / \mathrm{s}$ in BLA group mice. In the MOD group, the PWV was $3.7 \pm 0.9 \mathrm{~m} / \mathrm{s}$, which was significantly $(p<0.0001)$ different from the BLA group. The PWVs for mice in PCON group and SHXZF group were $1.6 \pm 1.1 \mathrm{~m} / \mathrm{s}$ and $1.8 \pm 1.1 \mathrm{~m} / \mathrm{s}$, respectively. The PWV in the PCON and SHXZF groups were also significantly $(p<0.001)$ different from the MOD group.

\subsection{Serum metabolite profiling by GC-MS}

A typical GC/MS total ion current (TIC) chromatogram of the Serum samples from the blank, model, and SHXZF-treat groups was illustrated in Figures 2(a), 2(b), and 2(c), respectively. It was hard to identify the ions that contributed to the discrimination of the SHXZF-treated and blank groups from thousands of signals using traditional statistical methods.In this study, the OPLS-DA method was applied to identify biomarkers that were related to AS development.

\subsection{Multivariate statistical analysis}

The abscissa $t[1]$ and ordinate $t[2]$ of the PCA score map represent the score values of each sample projected on the principal components PC1 and PC2, respectively. The obvious separation between the model and blank group suggests that significant serum metabolic disturbance occurred in the ApoE-/mice(Fig. 3A). We used OPLS-DA to further elucidate the differential variables. The two groups of samples have significant differences on the OPLS-DA score map (Fig. 3B). After 200 permutations were performed on the OPLS-DA model, the intercept values of R2 and Q2 are 0.994 and -0.009 . The negative 
value of Q2 intercept indicates the robustness of the model, which shows a low risk of overfitting and reliability (Fig. 3C).

\subsection{Identification of biomarkers and pathways}

To identify biomarkers in the model and blank group, the variables with VIP values above 1.0 were filtered out. Potential biomarkers were extracted from the loading plot (Fig.4), which were established based on OPLS-DA. The farther a point is away from the center, the more contribution it makes to the difference between the groups and the more likely it is to be a differential metabolite. According to VIP $>1$ (Huang et al. 2019), 96 difference variables were found. The names of a few metabolites were shown in Fig.4. 41 significantly changed metabolites could be identified as potential biomarkers and are listed in Table 2. Among them, 4 were decreased in model mice, whereas the other 37 were upregulated.

\subsection{Metabolomics in SHXZF intervention}

To evaluate the influence of SHXZF on serum metabolic profiles of the ApoE-/- mice, OPLS-DA scores plot was built to depict the general variation. The scores plot (Fig. 5A) showed that the SHXZF treatedgroup was much closer to the blank group, implying that SHXZF did effectively prevent the progression of AS and regulated the perturbed metabolism. By comparing the identified biomarker levels in the blank, model, and SHXZF-treated groups, the mean level of the 24 metabolites showed a tendency to normal at different degrees after taking SHXZF (Table 2). Among these metabolites, Palmitic acid and Oleic acid in the SHXZF-treated group were completely reversed to levels in the blank group (Table 2). It was revealed that the concentrations of these metabolites, which were altered in the mice of model group, had the tendency to come back from the model group to the blank group after taking SHXZF. Meanwhile, 8 metabolite-related pathways have been marked and identified through the MetaboAnalyst tool. According to the analysis results, biosynthesis of unsaturated fatty acids, lysine degradation, pentose phosphate pathway were significantly associated with effect of SHXZF on mice model of AS induced by high-fat diet (Fig. 5B).

\subsection{Differentially expressed proteins}

We used an TMT approach to identify proteins differentially expressed (DEPs) between different groups. The relative protein expression values were compared between groups (MOD vs. BLA and SHXZF vs. MOD) to identify the DEPs that were of great interest for their possible role in disease progression and the revelation of molecular drug targets. The threshold value was set as $<0.67$ and $>1.5$ fold change and $p<0.05$. Under this conditions, 125 proteins were found as DEPs between MOD and BLA. through which, 72 were down-regulated and 53 were up-regulated in MOD ApoE-/- mice. A total of 167 proteins were found as DEPs in the SHXZF-treated group and the MOD group, of which 129 proteins were significantly upregulated and 38 proteins were downregulated. There were 70 proteins (down in MOD and up in SHXZF, up in MOD and down in SHXZF ) identified as target proteins of SHXZF applied to AS mice by comparing to the proteins discussed above(Fig.6). 


\subsection{Bioinformatics analysis}

Bioinformatic analysis results are presented in Fig. 7. The cellular component analysis revealed that most of the DEPs belong to the extracellular space, extracellular region, extracellular exosome, mitochondrion, endoplasmic reticulum and mitochondrial inner membrane. Molecular functions mainly involve metal ion binding, hydrolase activity, calcium ion binding, oxidoreductase activity, serine-type peptidase activity, serine-type endopeptidase activity and iron ion binding. The biological process analysis indicated that these proteins play a role in 15 different biological processes, mainly involving oxidation-reduction process, cardiac muscle contraction and proteolysis. From pathway analysis, these proteins were shown to be mainly involved in metabolic pathways, the cAMP signaling pathway and myocardial contraction pathway. In addition, we use the STRING database to generate the DEP-related PPI network, as shown in Fig. 8.

\section{Discussion And Conclusion}

Metabonomics and proteomics are promising tools for studying the essence of TCM and mechanism of corresponding prescriptions, and provide scientific method on the efficacy of TCM (Zhang et al. 2017;Gou et al. 2019). In order to augment our understanding of the interventional mechanisms associated with the drug $邓$ the integration of metabolomic and proteomic as a reliable research method can be used to identify critical proteins and metabolites as well as the involved pathways that are affected by or respond to a drug in the body. Therefore, we investigated the alterations of protein and metabolic profiles in the serum and aortic arch tissue of $\mathrm{ApoE}^{-/-}$mice treated with SHXZF by using an integrated proteomics and metabolomics approach. In our research, 24 metabolites were identified as potential biomarkers while 70 proteins were found to be differentially expressed. Prior to this, we confirmed that SHXZF significantly ameliorates the histological features of high-fat diet-treated ApoE-/ -mice. The percentage of plaque area to the vascular lumen area results suggested that intake of SHXZF may be helpful for preventing and improving AS induced by high-fat diet. On the other hand, the SHXZF treatment group can significantly reduce PWV compared with the model group, thereby reducing the stiffness of the aorta.

In this study, we found that after treatment with SHXZF, the concentrations of several metabolites in the model group of mice tended to normal levels. As Fig.9 shows, SHXZF can regulate inflammation by interfering with lipid metabolism pathways. In addition, SHXZF can also slow down the process of AS by inhibiting the proliferation of vascular smooth muscle cells (VSMC) which are critical early events in AS and improving vascular stiffness. As we know, a major cell type in the vessel wall is represented by VSMC, which migrate from the media to intima in the early phase of AS development. The subsequent proliferation of VSMC in the intima and the concomitant phenotypic change from the contractile to the synthetic state are further features of VSMC in the progression of AS (Dzau et al. 2002; Sun et al. 2020; Jing et al. 2021).

\subsection{Lipid metabolism pathway}


According to the analysis, the related lipid metabolism pathway is mainly composed of biosynthesis of unsaturated fatty acids, lysine degradation and pentose phosphate pathway. The metabolic changes in serum, including oleic acid, palmitic acid and eicosapentaenoic acid, were mainly related to biosynthesis of unsaturated fatty acids. In mammals, polyunsaturated fatty acids (PUFAs) cannot be synthesized de novo, and therefore are essential fatty acids (EFAs). In addition, certain fatty acids function as signaling molecules and thus perform key biological functions, such as regulation of lipid metabolism and inflammation (Guillou et al. 2010). Oleic acid can be produced by palmitic acid under the action of $\Delta 9$ desaturase (OLE1). However, oleic acid is subsequently desaturated to linoleic acid. Fatty acid desaturases (Fads) converts linoleic acid to eicosapentaenoic acid by catalyzing the desaturation in the carbon backbone. Oleic acid is the fatty acid with the highest concentration in plasma, induces proliferation of VSMC and strongly enhances the growth-promoting activity of angiotensin II and endothelin 1 in VSMC. This mechanism is achieved by regulating the activation of NF-KB which regulates the expression of multiple inflammatory mediators, like IL-6, TNF-a and other factors involved in the metabolic alterations (Kern et al. 2001; Yun et al. 2006). Increased levels of proinflammatory cytokines such as IL- 6 and TNF-a, which are capable to activate NF-KB in different tissues to creat a cycle of selfmaintaining inflammation (Karin and Delhase 2000). Palmitic acid activates inflammatory signaling to produce numerous inflammatory cytokines in macrophages, including TNF-a and IL-6, promotes oxidized low-density lipoprotein uptake by macrophages (Wu et al. 2014). Palmitic acid also inhibits NO release by endothelial cells. As we all know, endothelial cells secrete the endothelium-derived relaxing factor nitric oxide, which inhibits the proliferation of vascular smooth muscle cells (Moers and Schrezenmeir 1997). Eicosapentaenoic acid restored the palmitic acid-mediated reduction of endothelial nitric oxide synthase (eNOS) and AMP-activated protein kinase (AMPK) phosphorylation..It can protect against atherogenesis via the AMPK/eNOS-related pathway (Lee et al. 2014). Eicosapentaenoic acid can also improve endothelial function and NO bioavailability (Mason et al. 2018). The metabolism pathway of lysine degradation mainly includes a-aminoadipic acid and saccharopine. The first step in the lysine degradation pathway is the formation of saccharopine and then a-aminoadipic acid, processes that are mitochondrial. L-lysine-2-ketoglutarate reductase (LKR) converts lysine to saccharopine by condensation with a-ketoglutarate. Accharopine dehydrogenase converts saccharopine to a-aminoadipic acid, producing a glutamate (Matthews 2020). A study have shown in a subgroup of 50 subjects, plasma glutamate levels correlated positively with plasma IL-6 concentrations. This finding supports the hypothesis that glutamate might have pro-inflammatory effects and thereby be involved in AS pathogenesis (Lehn-Stefan et al. 2021). The pentose phosphate pathway is a glucose turnover process which produces pentoses. It acts as essential parts of nucleotides. This path has two different stages. One is reversible non-oxidative phase in which phosphorylated sugars are converted to generate D-ribose. A study strongly suggest that D-ribose induced NF-KB inflammation, which may be a mechanism leading to AS (Hong et al. 2018).

\section{2 cAMP signaling pathway}

cAMP is a small molecule that relays into the cell the information carried by extracellular cue. It triggers the cascade of biochemical reactions required to achieve the appropriate cellular response to that specific 
stimulus. CAMP signaling pathway was activated in AS (Zhang et al. 2019). Natriuretic peptide precursorA (Nppa) is an early and specific marker for functional myocardium of the embryonic heart and it encodes for a precursor of atrial natriuretic peptide (ANP) (Song et al. 2015). ANP goes beyond a renal natriuretic hormone and also participates in vascular homeostasis. This function may possess novel antiatherogenic properties (Ichiki et al. 2013). By affecting the downstream cAMP signaling pathways, Nppa take part in AS. The cAMP acts as a crucial secondary messenger, involving in various biological processes (Chen et al. 2013). cAMP down-regulated the expression of NF-KB in endothelial cells. Its targets to increase cell apoptotic rate yet decrease cell proliferation rate of VSMC to suppress AS progression (Zhang et al. 2019).

\subsection{Myocardial contraction pathway}

The influence of myocardial contraction on arterial wall stress/strain (WS/S) and vascular stiffness is well recognized. Local increase of vessel stiffness resulting from wall-strain stiffening phenomenon may be critical in atherosclerotic lesions. The stiffness of conduit vessels can be estimated by PWV. The stiffer the vessel, the faster the pulse wave moving along the aorta. In this study, SHXZF was able to act on the myocardial contraction pathway, thus influencing the WS/S and vascular stiffness. Previous studies showed a significant correlation between increase of arterial wall stiffness in atherosclerotic mice and plaque development (Van Herck et al. 2009). Composed of RyR2, Atp2a, troponin and Myl2, the myocardial contraction pathway is an effective hemodynamic regulation system. The cardiac RyR2 mediates $\mathrm{Ca}^{2+}$ efflux from the sarcoplasmic reticulum (SR) to activate cardiac muscle contraction (Handhle et al. 2016). Its gene family has been known to play a role in the etiology of cardiovascular disease, including AS. The ATP2A genes encode for SERCA pumps regulate various cellular processes including cell growth. It has been shown that intracellular calcium changes through mechanotransduction signaling by endothelial cells (ECs) and surface concentration of ATP is believed to affect the calcium mobilization. Also, another key role of ATP is mediating the vasodilation and vascular tone. Both are related to AS (Wang et al. 2016). RyR2 and ATP2A affect the expression of troponins and Myl2 by affecting the cytoplasmic $\mathrm{Ca}^{2+}$ concentration, causing myocardial contraction. Myocardial contraction has a significant effect on local hemodynamics and wall shear stress (Meza et al. 2018). Various in vitro studies have shown that NO production is also elevated with higher wall shear stress magnitude. NO synthesis in areas of high wall shear stress appears to be a key mediator of atheroprotection through reducing endothelial permeability (Lehoux et al. 2006). In addition, related research have indicated that endothelial NO deficiency may contribute to vascular stiffness. The increase of NO bioavailability could contribute to a reduction in vascular stiffness (Fitch et al. 2006).

\section{Conclusion}

In this study, proteomics and metabolomics are integrated to evaluate the efficacy and mechanism of SHXZF in the treatment of AS. The complete analysis indicated that SHXZF could exert its therapeutic effect through three biological pathways: the CAMP signaling pathway, the lipid metabolism pathway and the myocardial contraction pathway. By interfering with lipid metabolism pathways to regulate 
inflammation, activating cAMP signaling pathway and myocardial contraction pathway to inhibit the proliferation of VSMC and improve vascular stiffness, and ultimately play an anti-atherosclerotic effect. Combinations of metabolomics and proteomics data can be employed in our future work, providing new ideas and methods for exploring the mechanism and role of traditional Chinese medicine in treating diseases.

\section{Abbreviations}

SHXZF: Shanhuaxiaozhi formulation; GC-MS: gas chromatography-mass spectrometry; TMT: Tandem Mass Tags; AS: Atherosclerosis; TCM: Traditional Chinese medicine; PWV: Pulse wave velocity; PCA: Principle component analysis; OPLS-DA : orthogonal partial least-squares-discriminant analysis;VIP: Variable importance in the projection; HCD: higher-energy collisional dissociation; KEGG: Kyoto Encyclopedia of Genes and Genomes; PPI: protein-protein interaction network; TIC: total ion current; DEPs: proteins differentially expressed; VSMC: vascular smooth muscle cells; PUFAs: polyunsaturated fatty acids; EFAs: essential fatty acids; eNOS: endothelial nitric oxide synthase; AMPK: AMP-activated protein kinase; LKR: L-lysine-2-ketoglutarate reductase; Nppa: Natriuretic peptide precursor-A; ANP: atrial natriuretic peptide; WS/S: wall stress/strain; SR: sarcoplasmic reticulum; ECs: endothelial cells

\section{Declarations}

\section{Ethics Approval and Consent to Participate}

Institutional ethics approval for the animal experiment was obtained from the Ethics Committee of Xiyuan Hospital.

\section{Consent for publication}

Not applicable.

\section{Availability of data and materials}

The datasets used and/or analysed are available from the corresponding author on reasonable request.

\section{Competing interests}

The authors declare that they have no conflicts of interest concerning this article.

\section{Funding}

Not applicable.

\section{Authors Contributions}


Ren Xing drafted the manuscript, Zhang Wantong was mainly responsible for interpreting the data, Gao Rui conceptualized the study and developed the first trial protocol. All other authors participated in the editing of the final manuscript and approved the final version.

\section{Acknowledgments}

This study was funded by the Ministry of Science and Technology of China (2017ZX09304003) and National Natural Science Foundation of China囚82004352】.

\section{References}

1. Chen ML, Yi L, Jin X, Liang XY, Zhou Y, Zhang T, Xie Q, Zhou X, Chang H, Fu YJ, Zhu JD, Zhang QY, Mi MT. Resveratrol attenuates vascular endothelial inflammation by inducing autophagy through the cAMP signaling pathway. Autophagy. 2013 Dec;9(12):2033-45.

2. Colvin KL, Yeager ME. Proteomics of pulmonary hypertension: could personalized profiles lead to personalized medicine? Proteomics Clin Appl. 2015 Feb;9(1-2):111 - 20.

3. Dzau VJ, Braun-Dullaeus RC, Sedding DG. Vascular proliferation and atherosclerosis: new perspectives and therapeutic strategies. Nat Med. 2002 Nov;8(11):1249-56.

4. Fitch RM, Rutledge JC, Wang YX, Powers AF, Tseng JL, Clary T, Rubanyi GM. Synergistic effect of angiotensin II and nitric oxide synthase inhibitor in increasing aortic stiffness in mice. Am J Physiol Heart Circ Physiol. 2006 Mar;290(3):H1190-8.

5. Gou XJ, Gao S, Chen L, Feng Q, Hu YY. A Metabolomic Study on the Intervention of Traditional Chinese Medicine Qushi Huayu Decoction on Rat Model of Fatty Liver Induced by High-Fat Diet. Biomed Res Int. 2019 Feb 7;2019:5920485.

6. Guillou H, Zadravec D, Martin PG, Jacobsson A. The key roles of elongases and desaturases in mammalian fatty acid metabolism: Insights from transgenic mice. Prog Lipid Res. 2010 Apr;49(2):186 - 99.

7. Huang B, Hu X, Wang J, Li P, Chen J. Study on chemical constituents of herbal formula Er Miao Wan and GC-MS based metabolomics approach to evaluate its therapeutic effects on hyperuricemic rats. J Chromatogr B Analyt Technol Biomed Life Sci. 2019 Jun 15;1118-1119:101-108.

8. Handhle A, Ormonde CE, Thomas NL, Bralesford C, Williams AJ, Lai FA, Zissimopoulos S. Calsequestrin interacts directly with the cardiac ryanodine receptor luminal domain. J Cell Sci. 2016 Nov 1;129(21):3983-3988.

9. Hong J, Wang X, Zhang N, Fu H, Li W. D-ribose induces nephropathy through RAGE-dependent NF-KB inflammation. Arch Pharm Res. 2018 Aug;41(8):838-847.

10. Ichiki T, Izumi R, Cataliotti A, Larsen AM, Sandberg SM, Burnett JC Jr. Endothelial permeability in vitro and in vivo: protective actions of ANP and omapatrilat in experimental atherosclerosis. Peptides. 2013 Oct; $48: 21-6$. 
11. Jing $Y, G a o B$, Han $Z$, Xia L, Xin S. The protective effect of HOXA5 on carotid atherosclerosis occurs by modulating the vascular smooth muscle cell phenotype. Mol Cell Endocrinol. 2021 Jun 11;534:111366.

12. Kern PA, Ranganathan S, Li C, Wood L, Ranganathan G. Adipose tissue tumor necrosis factor and interleukin-6 expression in human obesity and insulin resistance. Am J Physiol Endocrinol Metab. 2001 May;280(5):E745-51.

13. Karin M, Delhase M. The I kappa B kinase (IKK) and NF-kappa B: key elements of proinflammatory signalling. Semin Immunol. 2000 Feb;12(1):85-98.

14. Libby P, Buring JE, Badimon L, Hansson GK, Deanfield J, Bittencourt MS, Tokgözoğlu L, Lewis EF. Atherosclerosis. Nat Rev Dis Primers. 2019 Aug 16;5(1):56.

15. Lin HH, Charles AL, Hsieh CW, Lee YC, Ciou JY. Antioxidant effects of 14 Chinese traditional medicinal herbs against human low-density lipoprotein oxidation. J Tradit Complement Med. 2014 Nov 7;5(1):51 - 5 .

16. Lehoux S, Castier Y, Tedgui A. Molecular mechanisms of the vascular responses to haemodynamic forces. J Intern Med. 2006 Apr;259(4):381 - 92.

17. Luo Q, Li Z, Huang X, Yan J, Zhang S, Cai YZ. Lycium barbarum polysaccharides: Protective effects against heat-induced damage of rat testes and $\mathrm{H} 2 \mathrm{O} 2$-induced DNA damage in mouse testicular cells and beneficial effect on sexual behavior and reproductive function of hemicastrated rats. Life Sci. 2006 Jul 10;79(7):613 - 21.

18. Lee CH, Lee SD, Ou HC, Lai SC, Cheng YJ. Eicosapentaenoic acid protects against palmitic acidinduced endothelial dysfunction via activation of the AMPK/eNOS pathway. Int J Mol Sci. 2014 Jun 10;15(6):10334-49.

19. Lee AY, Park W, Kang TW, Cha MH, Chun JM. Network pharmacology-based prediction of active compounds and molecular targets in Yijin-Tang acting on hyperlipidaemia and atherosclerosis. $J$ Ethnopharmacol. 2018 Jul 15;221:151-159.

20. Lehn-Stefan A, Peter A, Machann J, Schick F, Randrianarisoa E, Heni M, Wagner R, Birkenfeld AL, Fritsche A, Häring HU, Staiger H, Stefan N. Elevated Circulating Glutamate Is Associated With Subclinical Atherosclerosis Independently of Established Risk Markers: A Cross-Sectional Study. J Clin Endocrinol Metab. 2021 Jan 23;106(2):e982-e989.

21. Leone S, Recinella L, Chiavaroli A, Orlando G, Ferrante C, Leporini L, Brunetti L, Menghini L. Phytotherapic use of the Crocus sativus L. (Saffron) and its potential applications: A brief overview. Phytother Res. 2018 Dec;32(12):2364-2375.

22. Liu X, Wu J, Tian R, Su S, Deng S, Meng X. Targeting foam cell formation and macrophage polarization in atherosclerosis: The Therapeutic potential of rhubarb. Biomed Pharmacother. 2020 Sep; 129:110433.

23. Li TT, Wang ZB, Li Y, Cao F, Yang BY, Kuang HX. The mechanisms of traditional Chinese medicine underlying the prevention and treatment of atherosclerosis. Chin J Nat Med. 2019 Jun;17(6):401412. 
24. Mason RP, Dawoud H, Jacob RF, Sherratt SCR, Malinski T. Eicosapentaenoic acid improves endothelial function and nitric oxide bioavailability in a manner that is enhanced in combination with a statin. Biomed Pharmacother. 2018 Jul;103:1231-1237.

25. Matthews DE. Review of Lysine Metabolism with a Focus on Humans. J Nutr. 2020 Oct 1;150(Suppl 1):2548S-2555S.

26. Meza D, Rubenstein DA, Yin W. A comprehensive fluid-structure interaction model of the left coronary artery. J Biomech Eng. 2018 Jul 5.

27. Moers A, Schrezenmeir J. Palmitic acid but not stearic acid inhibits NO-production in endothelial cells. Exp Clin Endocrinol Diabetes. 1997;105 Suppl 2:78-80.

28. Stavnsbo M, Resaland GK, Anderssen SA, Steene-Johannessen J, Domazet SL, Skrede T, Sardinha LB, Kriemler S, Ekelund U, Andersen LB, Aadland E. Reference values for cardiometabolic risk scores in children and adolescents: Suggesting a common standard. Atherosclerosis. 2018 Nov;278:299306.

29. Stone NJ, Robinson JG, Lichtenstein AH, Bairey Merz CN, Blum CB, Eckel RH, Goldberg AC, Gordon D, Levy D, Lloyd-Jones DM, McBride P, Schwartz JS, Shero ST, Smith SC Jr, Watson K, Wilson PW; American College of Cardiology/American Heart Association Task Force on Practice Guidelines. 2013 ACC/AHA guideline on the treatment of blood cholesterol to reduce atherosclerotic cardiovascular risk in adults: a report of the American College of Cardiology/American Heart Association Task Force on Practice Guidelines. J Am Coll Cardiol. 2014 Jul 1;63(25 Pt B):2889 - 934.

30. Song W, Wang H, Wu Q. Atrial natriuretic peptide in cardiovascular biology and disease (NPPA). Gene. 2015 Sep 10;569(1):1-6.

31. Sun D, Xiang G, Wang J, Li Y, Mei S, Ding H, Yan J. miRNA 146b-5p protects against atherosclerosis by inhibiting vascular smooth muscle cell proliferation and migration. Epigenomics. 2020 Dec;12(24):2189-2204.

32. Sun H, Zhang A, Yan G, Piao C, Li W, Sun C, Wu X, Li X, Chen Y, Wang X. Metabolomic analysis of key regulatory metabolites in hepatitis $C$ virus-infected tree shrews. Mol Cell Proteomics. 2013 Mar;12(3):710-9.

33. Van Herck JL, De Meyer GR, Martinet W, Van Hove CE, Foubert K, Theunis MH, Apers S, Bult H, Vrints CJ, Herman AG. Impaired fibrillin-1 function promotes features of plaque instability in apolipoprotein E-deficient mice. Circulation. 2009 Dec 15;120(24):2478-87.

34. Wang S, Chennupati R, Kaur H, Iring A, Wettschureck N, Offermanns S. Endothelial cation channel PIEZ01 controls blood pressure by mediating flow-induced ATP release. J Clin Invest. 2016 Dec $1 ; 126(12): 4527-4536$.

35. Wu D, Liu J, Pang X, Wang S, Zhao J, Zhang X, Feng L. Palmitic acid exerts pro-inflammatory effects on vascular smooth muscle cells by inducing the expression of C-reactive protein, inducible nitric oxide synthase and tumor necrosis factor-a. Int J Mol Med. 2014 Dec;34(6):1706-12.

36. Wang C, Niimi M, Watanabe T, Wang Y, Liang J, Fan J. Treatment of atherosclerosis by traditional Chinese medicine: Questions and quandaries. Atherosclerosis. 2018 Oct;277:136-144. 
37. Wu J, Ye X, Yang S, Yu H, Zhong L, Gong Q. Systems Pharmacology Study of the Anti-Liver Injury Mechanism of Citri Reticulatae Pericarpium. Front Pharmacol. 2021 Apr 12;12:618846.

38. Yun MR, Lee JY, Park HS, Heo HJ, Park JY, Bae SS, Hong KW, Sung SM, Kim CD. Oleic acid enhances vascular smooth muscle cell proliferation via phosphatidylinositol 3-kinase/Akt signaling pathway. Pharmacol Res. 2006 Aug;54(2):97-102.

39. Yang K, Zeng L, Ge A, Pan X, Bao T, Long Z, Tong Q, Yuan M, Zhu X, Ge J, Huang Z. Integrating systematic biological and proteomics strategies to explore the pharmacological mechanism of danshen yin modified on atherosclerosis. J Cell Mol Med. 2020 Dec;24(23):13876-13898.

40. Zhang L, Li T, Miao X, Ding L, Wang S, Wang Y. Aspirin suppresses NFKB1 expression and inactivates cAMP signaling pathway to treat atherosclerosis. Biofactors. 2019 May;45(3):343-354.

41. Zhang A, Zhou X, Zhao H, Zou S, Ma CW, Liu Q, Sun H, Liu L, Wang X. Metabolomics and proteomics technologies to explore the herbal preparation affecting metabolic disorders using high resolution mass spectrometry. Mol Biosyst. 2017 Jan 31;13(2):320-329.

\section{Tables}

Table 1 Composition of Shanhuaxiaozhi formulation (SHXZF).

\begin{tabular}{|lllll|}
\hline Latin name & Family & English name & Chinese name & Used part \\
\hline Crocus sativus L. & Iridaceae & Saffron & Zanghonghua & Stigmas \\
\hline Crataegus pinnatifida Bge. & Rosaceae & Crataegi Fructus & Shanzha & dried fruit \\
\hline $\begin{array}{l}\text { Citri Reticulatae Pericarpium. } \\
\text { Lycium barbarum L. }\end{array}$ & Rutaceae & Tangerine Peel & Chenpi & $\begin{array}{l}\text { dried } \\
\text { pericarp }\end{array}$ \\
\hline $\begin{array}{l}\text { Chrysanthemum morifolium } \\
\text { Ramat. }\end{array}$ & Solanaceae & Fructus Lycii & Gouqizi & fruit \\
\hline
\end{tabular}

Table 2 Significantly changed metabolites. The up or down arrows represent the relatively increased or decreased levels of the metabolites in model group or SHXZF group, respectively. a: compared to the blank group, b: compared to the model group. $* * \mathrm{p}<0.01, * \mathrm{p}<0.05, \# \mathrm{p}<0.05$. 


\begin{tabular}{|c|c|c|c|}
\hline NO. & Metabolites & MOD group ${ }^{a}$ & SHXZF group ${ }^{b}$ \\
\hline 1 & 2-monoolein & $\uparrow * \star$ & $\downarrow$ \\
\hline 2 & Beta-hydroxymyristic acid & $\downarrow^{\star *}$ & $\downarrow$ \\
\hline 3 & Citraconic acid & $\uparrow^{* *}$ & $\uparrow$ \\
\hline 4 & Lactose & $\uparrow^{\star *}$ & $\uparrow$ \\
\hline 5 & Palmitic acid & $\uparrow^{\star *}$ & $\downarrow \#$ \\
\hline 6 & Phytanic acid & $\uparrow^{\star * *}$ & $\downarrow$ \\
\hline 7 & Uric acid & $\uparrow * \star$ & $\uparrow$ \\
\hline 8 & Saccharopine & $\uparrow * \star$ & $\downarrow$ \\
\hline 9 & Maleimide & $\downarrow^{\star *}$ & $\downarrow$ \\
\hline 10 & Galactonic acid & $\uparrow * \star$ & $\downarrow$ \\
\hline 11 & Cis-gondoic acid & $\uparrow * \star$ & $\uparrow$ \\
\hline 12 & Conduritol-beta-epoxide & $\uparrow * \star$ & $\downarrow$ \\
\hline 13 & D-ribose & $\downarrow^{*}$ & $\uparrow$ \\
\hline 14 & Phenaceturic acid & $\uparrow^{*}$ & $\downarrow$ \\
\hline 15 & 1-hexadecanol & $\uparrow^{*}$ & $\uparrow$ \\
\hline 16 & Linolenic acid & $\uparrow^{*}$ & $\uparrow$ \\
\hline 17 & Quinic acid & $\uparrow^{*}$ & $\uparrow$ \\
\hline 18 & Guanine & $\uparrow^{*}$ & $\downarrow$ \\
\hline 19 & Alpha-tocopherol & $\uparrow^{*}$ & $\downarrow$ \\
\hline 20 & 6-phosphogluconic acid & $\uparrow^{*}$ & $\uparrow$ \\
\hline 21 & Xylonic acid & $\uparrow^{*}$ & $\downarrow$ \\
\hline 22 & Oleic acid & $\uparrow^{*}$ & $\downarrow \#$ \\
\hline 23 & Eicosapentaenoic acid & $\uparrow^{*}$ & $\downarrow$ \\
\hline 24 & Coniferin & $\uparrow^{*}$ & $\downarrow$ \\
\hline 25 & 3,6-anhydro-d-galactose & $\uparrow^{*}$ & $\downarrow$ \\
\hline 26 & 1,5-anhydroglucitol & $\uparrow^{*}$ & $\downarrow$ \\
\hline 27 & Alpha-aminoadipic acid & $\downarrow^{*}$ & $\uparrow$ \\
\hline 28 & L-kynurenine & $\uparrow^{*}$ & $\uparrow$ \\
\hline
\end{tabular}




\begin{tabular}{|llll|}
\hline 29 & 2-monopalmitin & $\uparrow^{*}$ & $\downarrow$ \\
\hline 30 & Isoheptadecanoic acid & $\uparrow^{*}$ & $\uparrow$ \\
31 & Octadecanol & $\uparrow^{*}$ & $\uparrow$ \\
32 & Elaidic acid & $\uparrow^{*}$ & $\uparrow$ \\
\hline 33 & Isoxanthopterin & $\uparrow^{*}$ & $\downarrow$ \\
\hline 34 & Dl-dopa & $\uparrow^{*}$ & $\downarrow$ \\
\hline 35 & L-alanine-alanine & $\uparrow^{*}$ & $\uparrow$ \\
\hline 36 & D-fructose 2,6-bisphosphate & $\uparrow^{*}$ & $\uparrow$ \\
\hline 37 & 2-deoxytetronic acid & $\uparrow^{*}$ & $\downarrow$ \\
\hline 38 & Dehydroascorbic acid & $\uparrow^{*}$ & $\downarrow$ \\
\hline 39 & Glycerol 3-phosphate & $\uparrow^{*}$ & $\uparrow$ \\
\hline 40 & Isolinoleic acid & $\uparrow^{*}$ & $\downarrow$ \\
\hline 41 & N-acetyl-d-hexosamine & $\uparrow^{*}$ & $\downarrow$ \\
\hline
\end{tabular}

Figures 
A

BLA
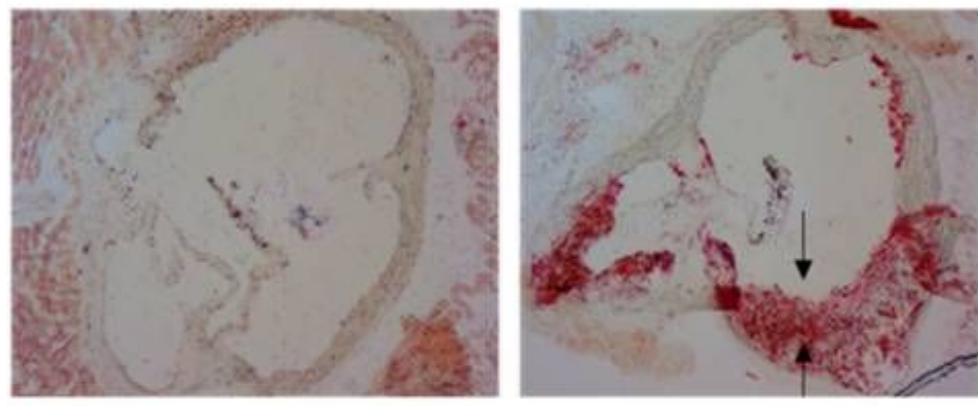

B

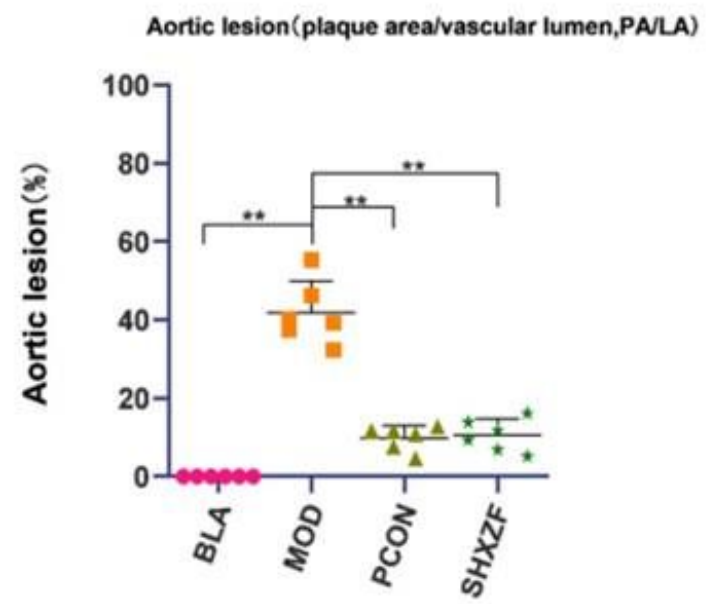

C

PWV(distance divided by pulse wave propagation time)

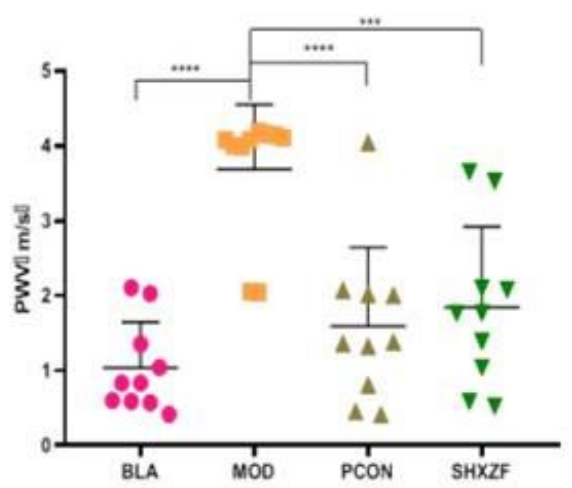

\section{Figure 1}

Effect of SHXZF on the atherosclerotic plaque changes in ApoE-/- AS mice. Oil red O staining of the aortic sinus $(A, n=6)$. The arrow shows the area where atherosclerotic plaque is formed. The statistical analysis of atherosclerotic plaque in the aortic sinus was shown in (B). Data was presented as mean \pm SD. Statistic difference is indicated as **p $<0.01$ vs BLA group. ** $<<0.01$, vs MOD group. The PWV of the aortic was measured in each group in (C). Statistic difference is indicated as $* \star \star \star p ~<0.0001$ vs BLA group. ${ }^{* \star *} p<0.001$, vs MOD group. 


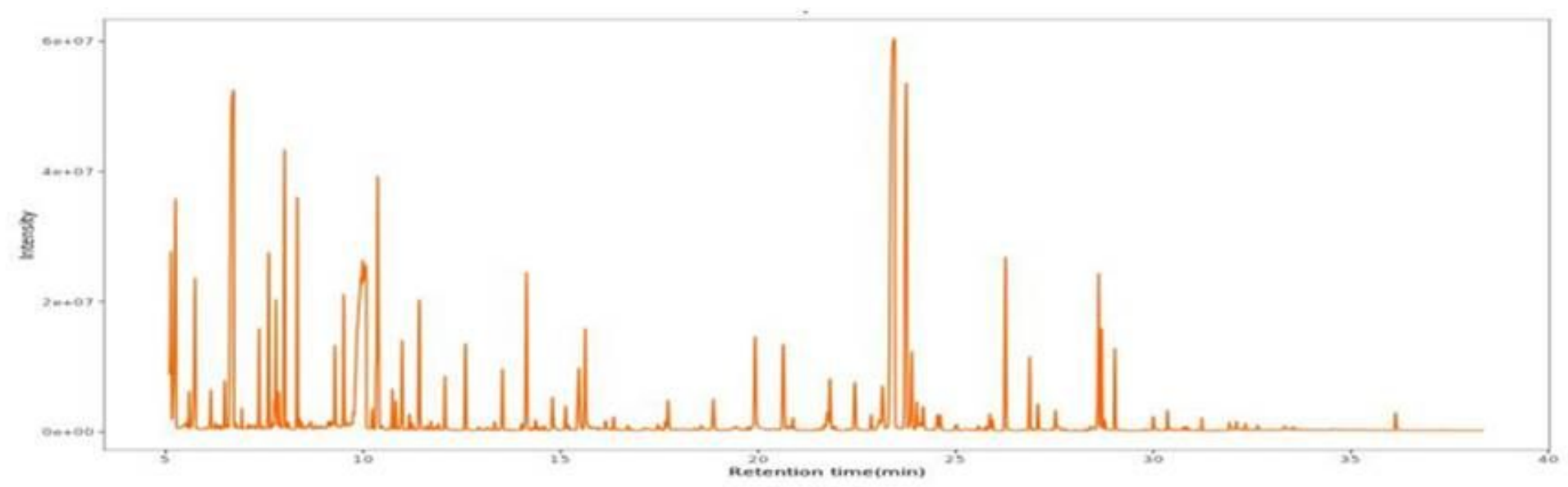

(a)
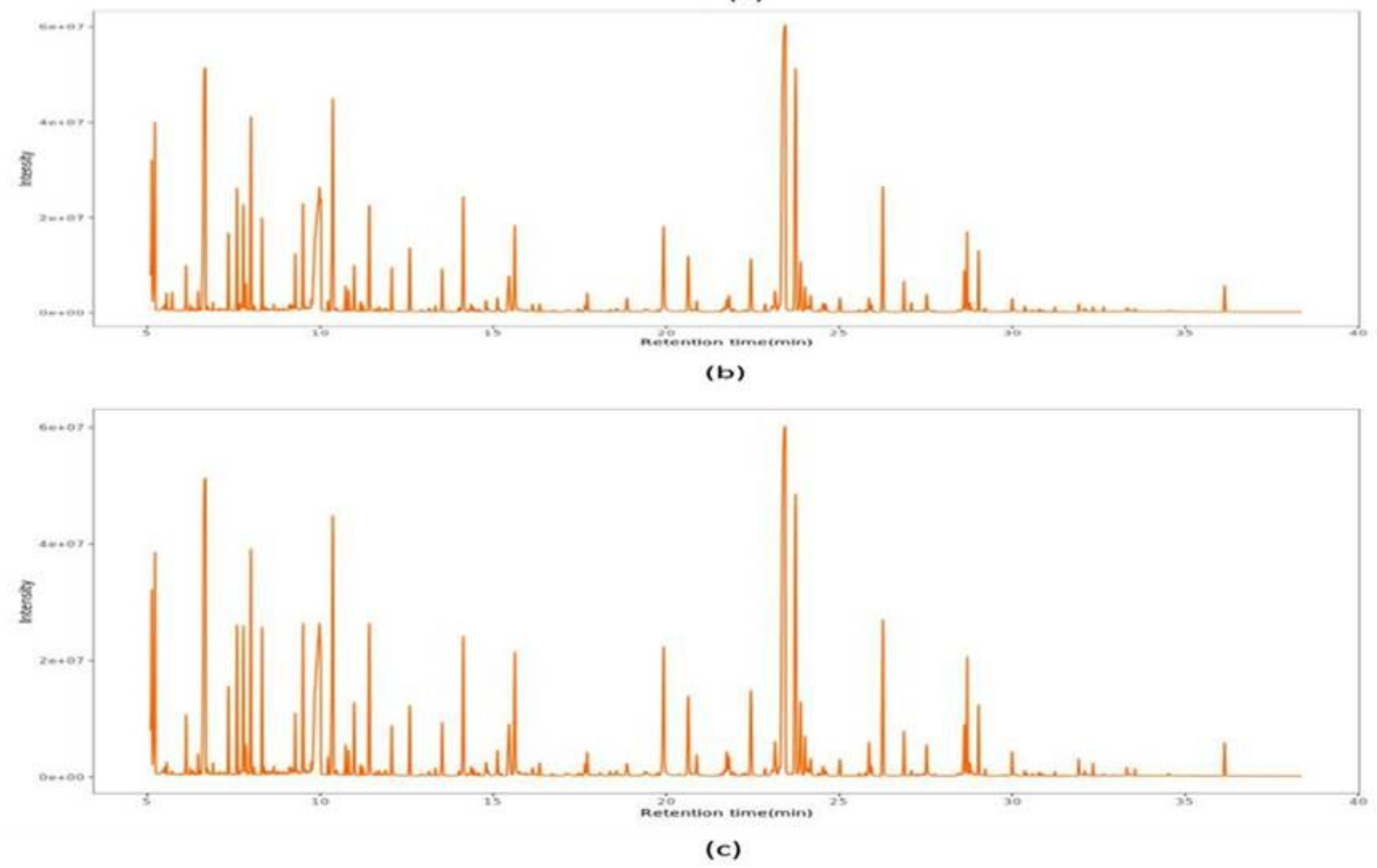

Figure 2

Typical GC/MS spectra of urine samples from (a) blank group, (b) model group, and (c) SHXZF-treat group 
A

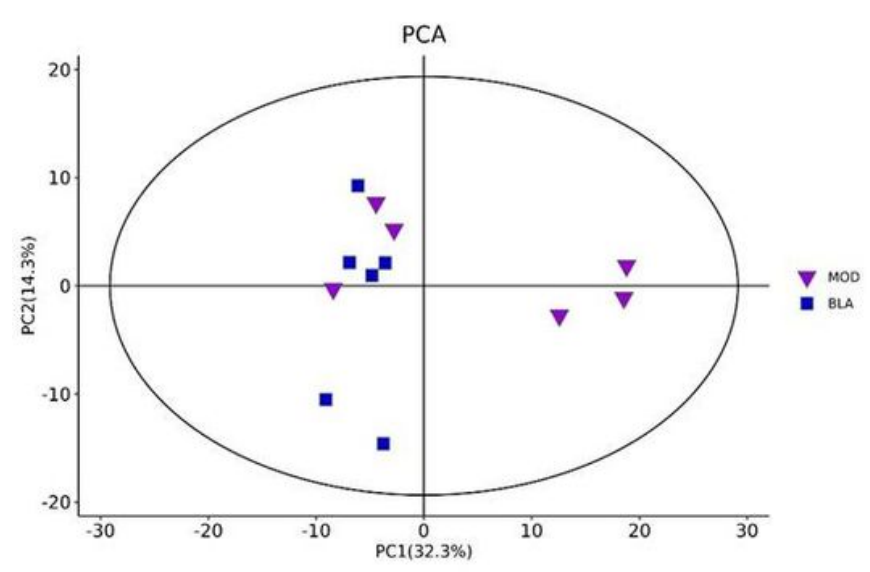

B

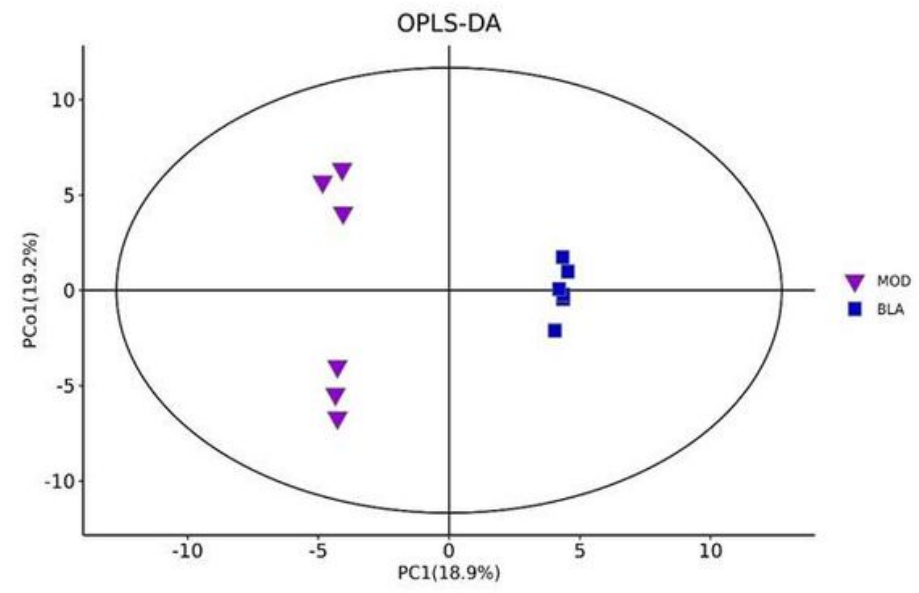

C

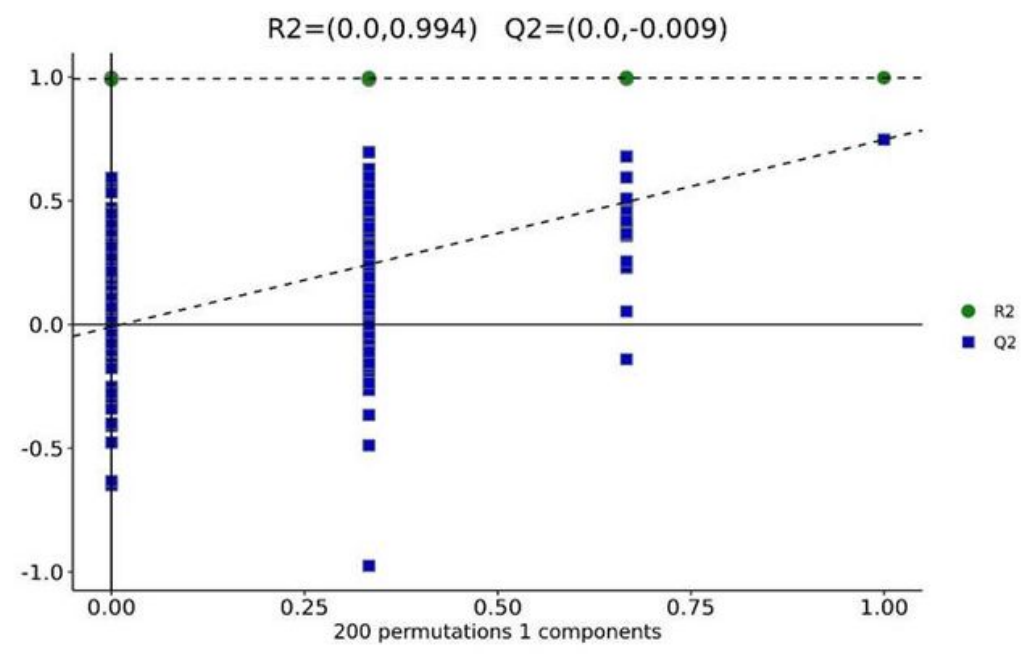

Figure 3

Score plot of blank group (blue square) and modle group (purple triangle) from a PCA modle (A) and a OPLS-DA modle (B). Two hundred permutations were performed, and the resulting R2 and Q2 values were plotted. Green circle: R2; blue square: Q2. The green dotted line represents the regression line for R2 and the blue dotted line for Q2. 


\section{Loading-OPLS-DA}

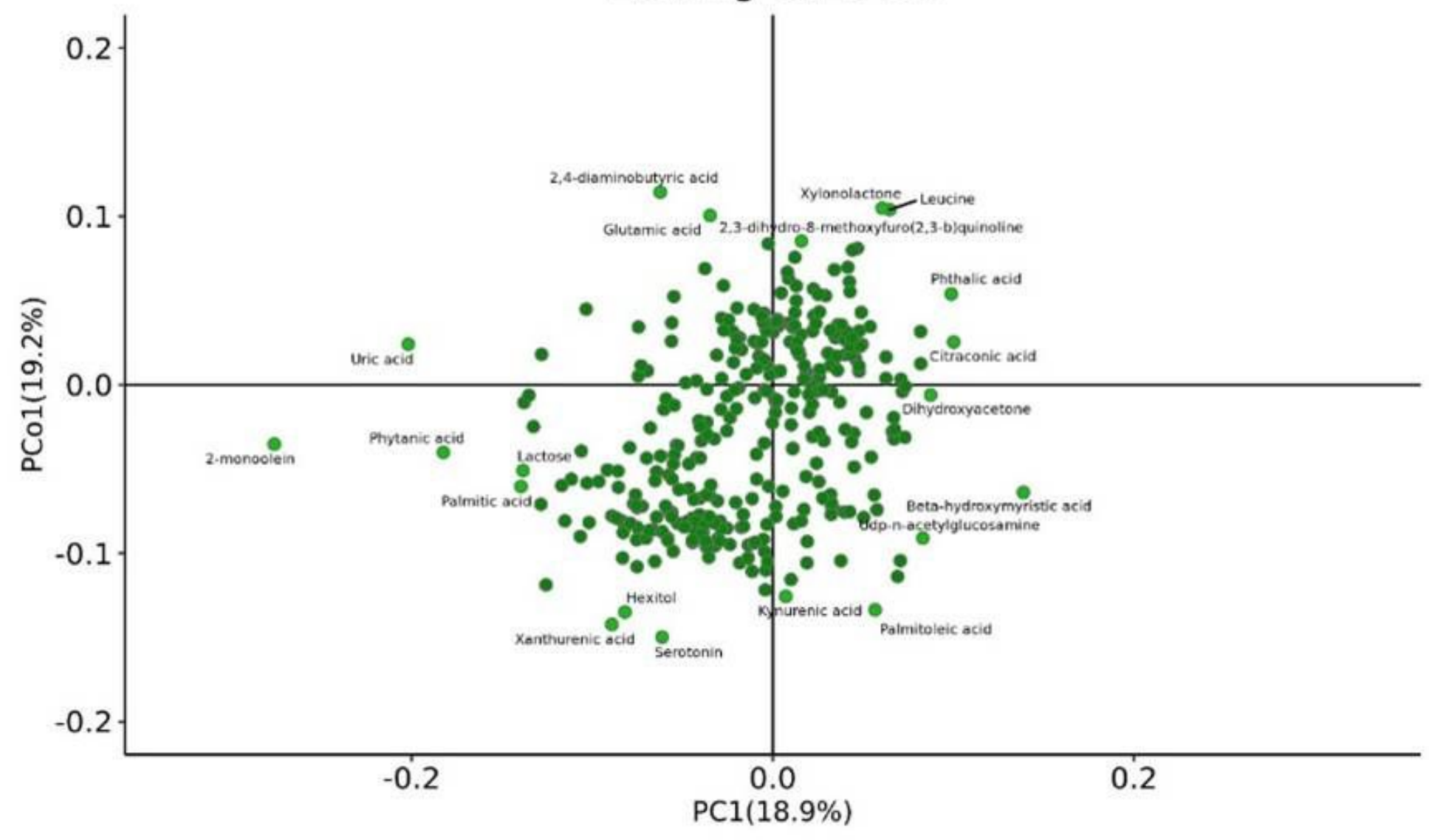

Figure 4

Loading plot from OPLS-DA model classifying model obtained from blank group and model group.

A

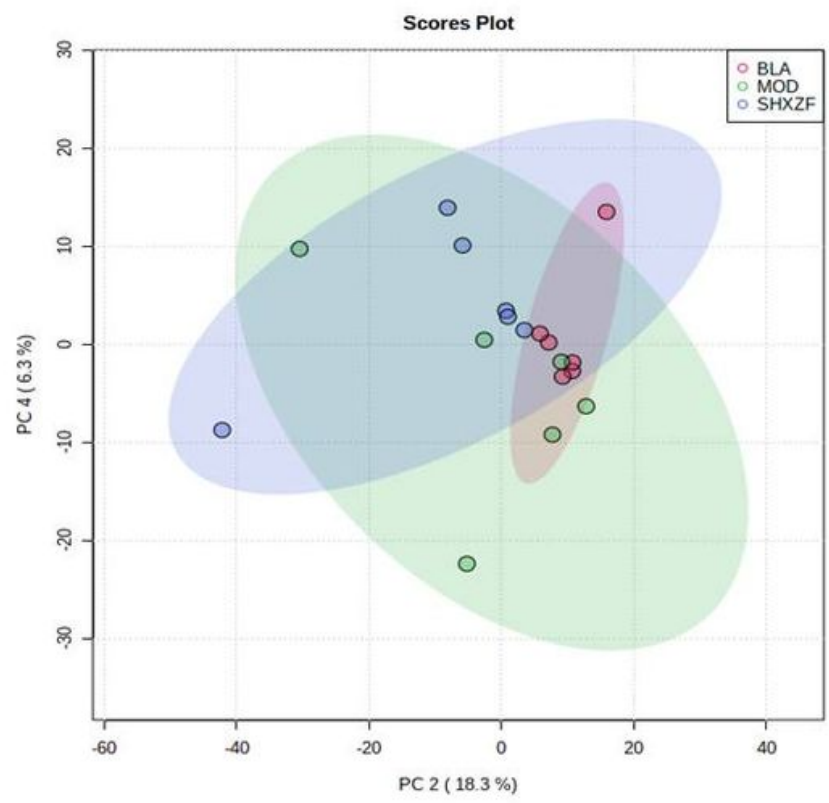

B

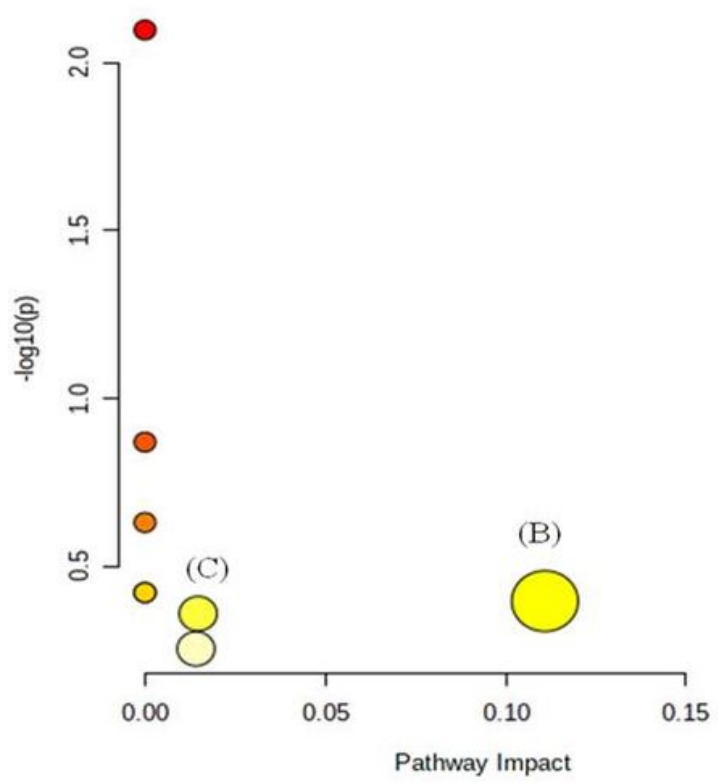

(A)

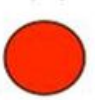




\section{Figure 5}

Score plot of PLS-DA model obtained from blank group (red circle), model group (green circle), and SHXZF-treat group (blue circle). Summary of pathway associated with effect of SHXZF on ApoE-/- mice of AS. (A) biosynthesis of unsaturated fatty acids, (B) lysine degradation, (C) pentose phosphate pathway.

\section{DOWN IN SHXZF DOWN IN MOD}

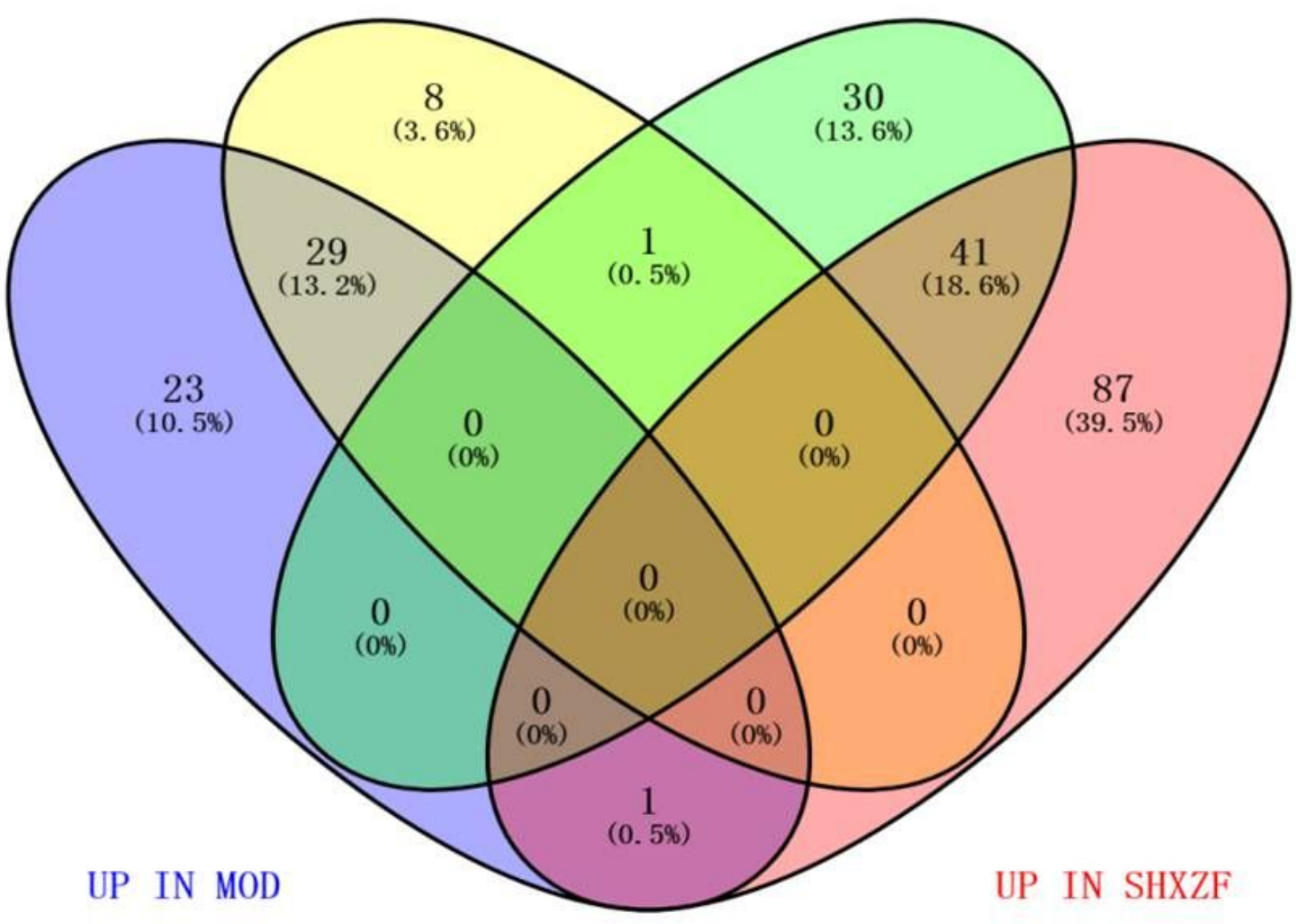

\section{Figure 6}

Venn diagrams showing the distribution of shared DEPs. Blue represents upregulated proteins in the model group (up in MOD), green represents downregulated proteins in the model group (down in MOD), 
pink represents upregulated proteins in the SHXZF-treat group (up in SHXZF), and yellow represents downregulated proteins in the SHXZF-treat (down in SHXZF). Overlapping sections indicate the number of common proteins across different groups.

A

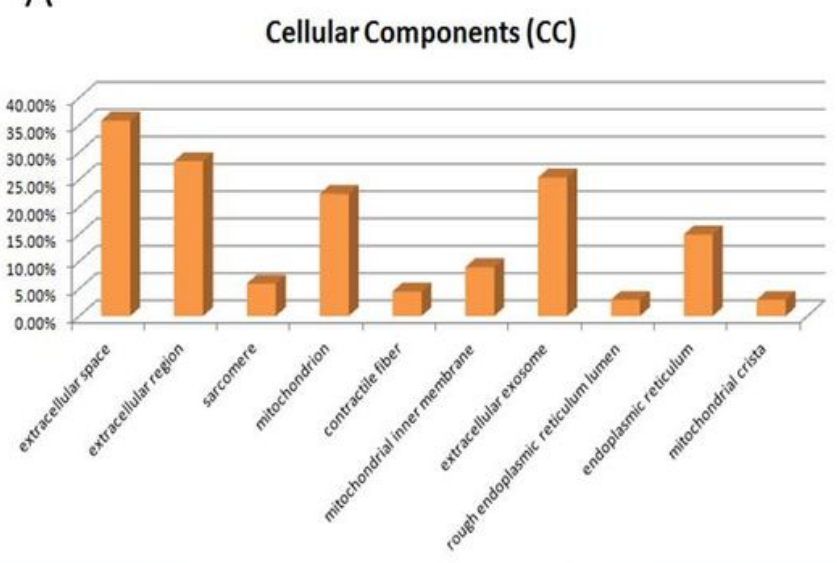

B

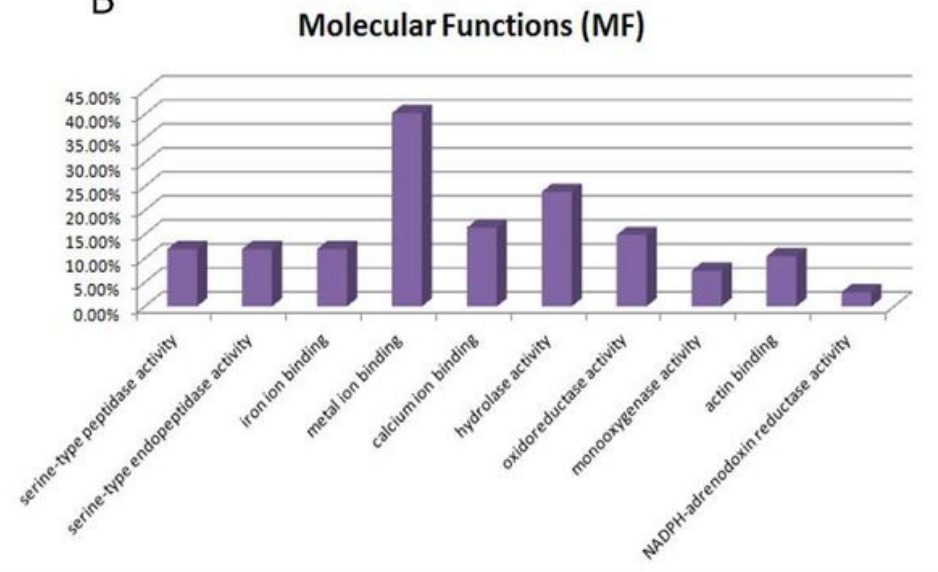

C

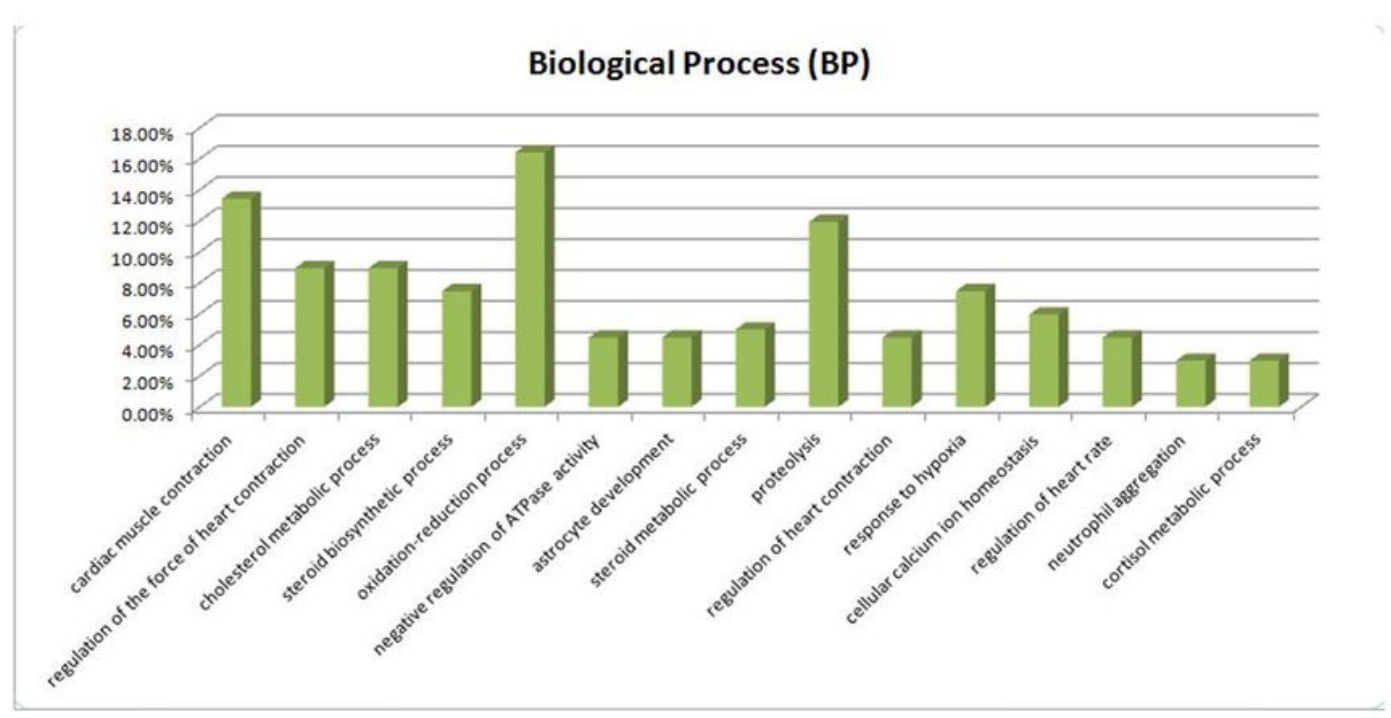

Figure 7

The proportion of the differentially expressed proteins categorized by function. A: cellular components; $\mathrm{B}$ : molecular function; C: biological processes 


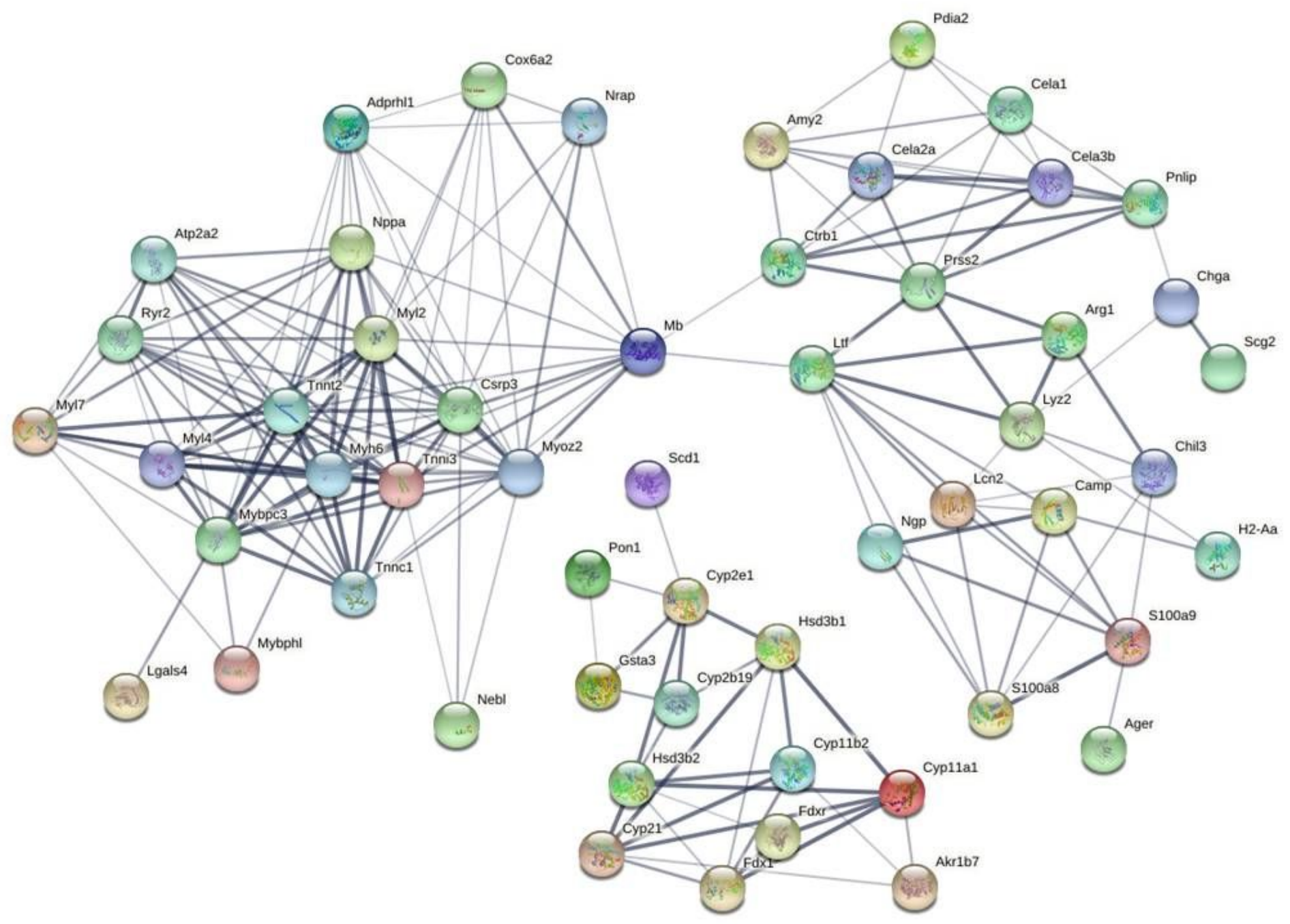

Figure 8

Protein-protein interaction networks analysis of the differentially expressed proteins with the STRING. The circles represent proteins while the straight lines represent the interactions between different proteins. 


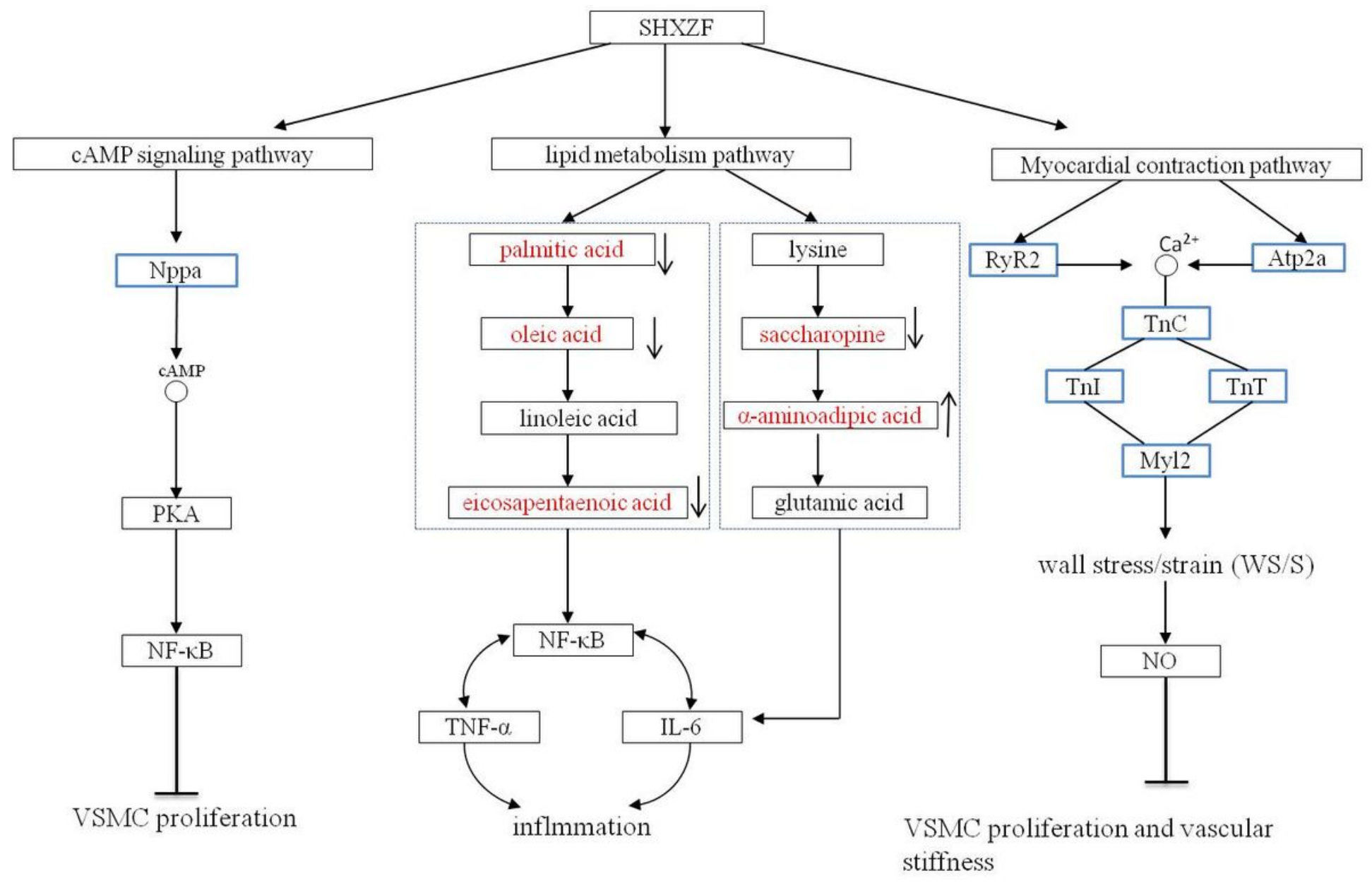

\section{Figure 9}

The perturbed metabolites, proteins and corresponding metabolic pathways related to SHXZF treatment by integrating the proteome and metabolome data sets. Elevation (up thin arrows) and reduction (down thin arrows) of the levels of metabolites were indicated. The metabolites were presented with red texts and the proteins were circled by blue box.

\section{Supplementary Files}

This is a list of supplementary files associated with this preprint. Click to download.

- Graphicalabstract.png 